\title{
Use of static and dynamic 18F-FDopa PET parameters for detecting patients with glioma recurrence or progression
}

\section{Timothée Zaragori}

Université de Lorraine

Merwan Ginet

Université de Lorraine, CHRU-Nancy

Pierre-Yves Marie

Université de Lorraine, CHRU-Nancy

Veronique Roch

Université de Lorraine CHRU-Nancy

Rachel Grignon

Université de Lorraine CHRU Nancy

Guillaume Gauchotte

Université de Lorraine CHRU Nancy

Fabien Rech

Université de Lorraine CHRU Nancy

Marie Blonski

Université de Lorraine CHRU Nancy

Zohra Lamiral

Université de Lorraine CHRU Nancy

Luc Taillandier

Université de Lorraine CHRU Nancy

Laetitia Imbert

Université de Lorraine CHRU Nancy

Antoine Verger ( $\nabla$ a.verger@chru-nancy.fr)

CHRU Nancy https://orcid.org/0000-0003-2194-0328

Original research

Keywords: 18F-FDopa PET, dynamic analysis, glioma, recurrence, WHO 2016 classification

Posted Date: March 13th, 2020

DOI: https://doi.org/10.21203/rs.3.rs-17078/v1 
License: (c) (i) This work is licensed under a Creative Commons Attribution 4.0 International License. Read Full License 


\section{Abstract}

Background: Static 18 F-FDopa PET images are currently used for identifying patients with glioma recurrence/progression after treatment, although the additional diagnostic value of dynamic parameters remains unknown in this setting. The aim of the present study was to evaluate the performances of static and dynamic 18 F-FDopa PET parameters for detecting patients with glioma recurrence/progression as well as to assess further relationships with patient outcome. Fifty-one consecutive patients who underwent an 18 F-FDopa PET for a suspected glioma recurrence/progression at post-resection MRI, were retrospectively included. Static parameters including mean and maximum tumor-to-normal-brain (TBR), tumor-to-striatum (TSR) ratios, and metabolic tumor volume (MTV), as well as dynamic parameters with time-to-peak (TTP) values and curve slope, were tested for predicting: 1) glioma recurrence/progression at 6-months after the PET exam and 2) survival on longer follow-up.

Results: All static parameters were significant predictors of a glioma recurrence/progression (accuracy $\geq 94 \%$ ) with all parameters also associated with mean progression-free survival (PFS) in the overall population (all $p<0.001,29.7$ vs. 0.4 months for TBR max , TSR max and MTV). The curve slope was the sole dynamic PET predictor of glioma recurrence/progression (accuracy $=76.5 \%$ ) and was also associated with the mean PFS ( $p<0.001,18.0$ vs. 0.4 months). However, no additional information was provided relative to static parameters in multivariate analysis.

Conclusion: Although patients with glioma recurrence/progression can be detected by both static and dynamic 18 F-FDopa PET parameters, most of this diagnostic information can be achieved by conventional static parameters.

\section{Background}

Amino-acid PET has been proposed as a criterion for detecting glioma recurrence by the Response Assessment in Neuro-Oncology (RANO) group [1] and has become a current indication for differentiating glioma recurrence from treatment-induced changes, as stated in the recent joint European Association of Nuclear Medicine (EANM)/European Association of Neuro-oncology (EANO)/RANO practice guidelines [2].

${ }^{18} \mathrm{~F}$-FDopa PET is an amino acid PET tracer for which the ability to diagnose glioma recurrence has been established [3-5]. In particular, ${ }^{18}$ F-FDopa PET was found to detect glioma recurrence with an accuracy as high as $82 \%$ in a retrospective study of 110 patients, in which the lesion-to-normal-tissue ratio was additionally predictive of progression-free survival (PFS) [6]. More recently, in a study in which glioma recurrence has been proven histologically, ${ }^{18} \mathrm{~F}$-FDopa PET accuracy was reported to reach $84 \%$, using maximum ${ }^{18} \mathrm{~F}$-FDopa uptake as diagnostic parameter [7]. This accuracy for detecting glioma recurrence was even higher, reaching $96 \%$, in a prospective albeit smaller study of 28 patients [8].

However, all of the aforementioned ${ }^{18} \mathrm{~F}$-FDopa PET studies [3-8] were conducted while solely taking into account ${ }^{18} \mathrm{~F}$-FDopa PET static parameters whereas information provided by dynamic parameters was not 
considered. ${ }^{18} \mathrm{~F}$-FDopa dynamic parameters were nonetheless assessed to distinguish high grade from low grade recurrent gliomas regards to the World Health Organization (WHO) 2007 classification [9]. Interestingly, such dynamic parameters have shown more recently, through the WHO 2016, classification that their integration enhances the diagnostic information provided by ${ }^{18} \mathrm{~F}$-FDopa PET in a series of newly-diagnosed gliomas [10]. However, it remains unknown whether they may also enhance the diagnostic accuracy of ${ }^{18}$ F-FDopa PET prescribed for detecting glioma recurrence/progression.

The aim of the present study was thus to assess the performances of ${ }^{18} \mathrm{~F}$-Dopa PET with both static and dynamic parameters, for detecting patients with progressive or recurrent glioma and for assessing further relationships with patient outcome.

\section{Materials And Methods}

\section{Patients}

From October 2012 to October 2017, 245 patients referred to the Department of Nuclear Medicine at the CHRU Nancy for brain tumor assessment were investigated by ${ }^{18}$ F-FDopa PET. Fifty-one of these patients were retrospectively selected on the basis that they had an initial history of surgically-resected glioma and that the considered ${ }^{18} \mathrm{~F}$-FDopa PET had been prescribed for differentiating recurrence/tumor progression from post-therapeutic effects after a non-contributive MRI. As usual in our department, a minimum delay of 2 months is always respected between the surgery or the end of the radiotherapy and performing ${ }^{18} \mathrm{~F}$-FDopa PET in order to reduce the risk of ${ }^{18} \mathrm{~F}$-FDopa PET false positives [11].

In such patients, a clinical follow-up and MRI are systematically planned at least every 3 months or at shorter intervals as clinically indicated after surgery [6]. The final diagnosis of glioma recurrence/progression at 6 month or for further assessment of survival was blinded from the ${ }^{18} \mathrm{~F}$-FDopa PET results and based on current guidelines for which a recurrence/progression is the result of any new tumor brain lesion at MRI and/or clear increase in tumor size or in contrast enhancement, and/or significant clinical deterioration, with all of these criteria not being attributable to other non-tumor causes and not due to steroid decrease [12-14]. Patients with lesions which were classified as therapy-related changes due to the course of the disease did not receive any additional therapy beyond the standard therapy due to suspected recurrence. Neuropathological data were additionally used for this diagnosis in 4 patients having undergone stereotactic biopsy or open resection.

The assessment of ${ }^{18} \mathrm{~F}$-FDopa PET parameters for differentiating recurrence/progression from posttherapeutic effects was based on the evaluation of the previous criteria during a 6 months follow-up period. However, PFS and overall survival (OS) were calculated from the date of the PET exam to the date of definite diagnosis of progression and of death, respectively, with a minimum delay of 19 months of observation. The final date for reporting any event for PFS or OS was June 1st, 2019. 
The local ethics committee (Comité d'Ethique du CHRU de Nancy) approved the retrospective data evaluation on June 7, 2018, and authorization from the CNIL (National Commission on Information Technology and Civil Liberties) was delivered on June 25, 2018 (authorization $n^{\circ}$ R2018-11). This study complied with the principles of the Declaration of Helsinki. Informed consent was obtained from all individuals included in the study.

\section{PET recordings and image reconstruction}

${ }^{18} \mathrm{~F}$-FDopa PET-computed tomography (CT) scans were obtained on a Biograph hybrid system involving a six-detector CT for attenuation correction (Biograph 6 True Point, SIEMENS, Erlangen, Germany). Patients were instructed to fast for at least 4 hours with certain patients also receiving Carbidopa administration one hour prior to their exam. The CT scan was recorded first and immediately followed by a $30 \mathrm{~min} 3 \mathrm{D}$ list-mode PET recording initiated during the bolus injection of $3 \mathrm{MBq}$ of ${ }^{18} \mathrm{~F}$-FDopa per kilogram of body weight. The static PET images were reconstructed from the list mode data ranging from 10 to $30 \mathrm{~min}$ post-injection $[2,5]$ while the PET images reconstructed for dynamic parameters encompassed 6 consecutive frames of $20 \mathrm{sec}$ each followed by 28 frames of 1 min each [15].

All static and dynamic images were reconstructed with an OSEM 2D algorithm ( 2 iterations, 21 subsets, 4$\mathrm{mm}$ Gaussian post-reconstruction filter), corrected for attenuation, scatter and radioactive decay, and displayed in a $256 \times 256$ matrix with $2.7 \times 2.7 \times 3.0 \mathrm{~mm}^{3}$ voxels.

\section{Analyses of PET images}

Regions of interest (ROIs) were placed on the static PET images using a dedicated software (DOSIsoft, Cachan, France). A spherical ROI of $2 \mathrm{~cm}$ diameter, centered on the maximum lesion uptake, was used for determining maximum and mean Standardized Uptake Values (SUV $\mathrm{max}_{\max }$ and SUV $\mathrm{V}_{\text {mean }}$, respectively). Tumor-to-striatum (TSR) and tumor-to-normal-brain (TBR) ratios were computed as SUV mean or SUV max $_{\text {max }}$ of the lesion uptake divided by the $S U V_{\text {mean }}$ of the striatum $\left(\mathrm{TSR}_{\text {mean }}\right.$ and $T S R_{\text {max }}$ ) or of normal brain $\left(T B R_{\text {mean }}\right.$ and $\left.T B R_{\text {max }}\right)$. The $S U V_{\text {mean }}$ from the striatum was obtained from the contralateral basal ganglia, which was segmented using a threshold of $65 \%$ of maximal uptake, while the normal reference

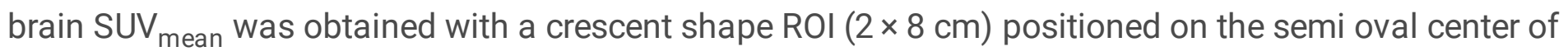
the unaffected contralateral hemisphere, including white and grey matter [2].

When no abnormal ${ }^{18} \mathrm{~F}$-FDopa uptake was detected, the ROIs of the potential residual tumor were placed at the site of maximal MRI abnormalities with a fused display of PET and Fluid Attenuation Inversion Recovery (FLAIR) MRI images [5].

As previously described [16], the metabolic tumor volume (MTV) was obtained through a 3D autocontouring process with a threshold corresponding to the SUV $\mathrm{V}_{\text {mean }}$ of the contralateral striatum.

In addition, time-activity curves, representing the evolution of the $\mathrm{TBR}_{\text {mean }}$ as a function of time $\left(\mathrm{TAC}_{\text {ratio }}\right)$, were extracted with the PLANET ${ }^{\circledR}$ Dose software (DOSIsoft, Cachan, France) and with the ROIs 
previously placed on static images (see above). Each dynamic frame was previously registered on the CT images, in order to take into account potential patient movements during acquisitions [17]. Two dynamic parameters were determined from fitted curves to overcome noise effects, using a method already validated for ${ }^{18}$ F-FET in the same setting [18], namely: i) Time-To-Peak (TTP), corresponding to the delay between the onset of the dynamic acquisition (time of tracer injection) and the time-point of the maximal $\mathrm{TBR}_{\text {mean }}$ value, and ii) slope, calculated with a linear regression applied from the 10th to 30th minute.

\section{Statistical analysis}

Categorical variables are expressed as percentages and continuous variables as median and interquartile range due to the non-normality of variable distributions. Among the considered continuous variables, those showing a p-value lower than 0.05 for the Mann-Whitney tests applied between patients with glioma recurrence/progression and the remaining patients, were considered as univariate predictors.

A multivariable logistic regression model including parameters with a $p$-value $<0.1$ in univariable analysis, was performed for predicting patients with glioma recurrence/progression. The optimal threshold for each static and dynamic PET parameters was determined from ROC curves using the maximal value of the product of sensitivity and specificity.

The dichotomized parameters, which were determined according to these optimal thresholds, were additionally used in survival analyses. For this purpose, survival probabilities were calculated according to the Kaplan-Meier method, with the log-rank test used to compare survival curves. Analyses were performed with SPSS (SPSS Statistics for Windows, Version 20.0. Armonk, NY: IBM Corp).

P-values obtained in univariate analysis as well as in survival analysis were adjusted using BenjaminiHochberg correction in order to reduce the risk of false discovery [19].

\section{Results}

\section{Patient characteristics and follow-up data}

The study population included 51 patients with a median age of 50.8 [44.4-59.0] years, 23 of whom were women. According to the 2016 WHO classification for gliomas [20], 8 gliomas (16\%) had been initially classified as IDH-mutant astrocytomas (including 2 with anaplastic component), 6 (12\%) as IDH-wildtype astrocytomas (including 2 with anaplastic component), 12 (24\%) as IDH-mutant and 1p/19q co-deleted oligodendrogliomas (including 4 with anaplastic component), 22 (43\%) as IDH-wildtype glioblastomas (GBM) and $3(6 \%)$ as IDH-mutant GBM. Median delay times from the date of surgery to the ${ }^{18} \mathrm{~F}$-FDopa PET exam and from the date of the non-contributive MRI to the PET exam were 12.7 [5.9-23.5] months and $16[7-30]$ days, respectively.

After the subsequent 6-month follow-up period, 34 patients were ultimately considered as having a recurrent or progressive glioma (3 IDH-mutant astrocytomas, 6 IDH-wildtype astrocytomas, 6 IDH-mutant 
and 1p/19q co-deleted oligodendrogliomas, 17 IDH-wildtype GBM and 2 IDH-mutant GBM) with 4 cases exhibiting a second recurrence and 2 cases a third one. The remaining 17 patients were thus considered to exhibit only treatment-related changes at MRI. Patient characteristics are detailed in Table 1 as well as in the supplemental Table.

Table 1

Patient characteristics

\begin{tabular}{|ll|}
\hline $\mathbf{n}=\mathbf{5 1}$ & Value \\
\hline Age (years) & \\
\hline Median [Range] & $50.8[21.2 ; 75.3]$ \\
\hline Female gender $\mathrm{n}(\%)$ & $23(45)$ \\
\hline Primary histopathological type $\mathrm{n}(\%)$ & $8(16)$ \\
\hline IDH-mutant astrocytoma & $6(12)$ \\
\hline IDH-wildtype astrocytoma & $12(24)$ \\
\hline IDH-mutant and 1p/19q co-deleted oligodendroglioma & $22(43)$ \\
\hline IDH-wildtype glioblastoma & $3(6)$ \\
\hline IDH-mutant glioblastoma & \\
\hline Histopathological WHO grade of the primary tumor $\mathrm{n}(\%)$ & $18(35)$ \\
\hline II & $8(16)$ \\
\hline III & $25(49)$ \\
\hline IV & $25(49)$ \\
\hline Primary treatment $\mathrm{n}(\%)$ & $15(29)$ \\
\hline Surgery & $2(18)$ \\
\hline Surgery + radiotherapy & 250 (4) \\
\hline Surgery + chemotherapy & \\
\hline Surgery + radiotherapy + chemotherapy & \\
\hline WHO: World Health Organization & \\
\hline
\end{tabular}

During an observation period of 41 [23-50] months following the PET exam, 22 patients (43\%) died and $43(84 \%)$ had evidence of tumor progression. In the overall population, median survival was 24 [14-43] months.

\section{PET prediction of glioma recurrence/progression}


As detailed in Tables 2 and 3, all studied PET parameters, except TTP, were significant univariate predictors of glioma recurrence/progression (all adjusted $p<0.001$ ), with a global diagnostic accuracy of $96 \%$ being reached with $\mathrm{TBR}_{\max }, \mathrm{TSR}_{\max }$ and MTV. Meanwhile, the curve slope was the sole significant dynamic PET predictor, although its predictive value was somewhat lower than that obtained with the other PET predictors (-i.e. with a lower area under the ROC curve and with a global diagnostic accuracy of only $76.5 \%$, as detailed in Table 3 ).

Table 2

Median [interquartile range] of PET parameters in the overall population as well as comparatively between the 2 groups of patients with or without glioma recurrence or progression.

\begin{tabular}{|c|c|c|c|c|}
\hline Parameter & Overall & $\begin{array}{l}\text { No } \\
\text { Recurrence/progression } \\
(n=17)\end{array}$ & $\begin{array}{l}\text { Recurrence/ progression } \\
(n=34)\end{array}$ & $\begin{array}{l}\text { Adjusted } \\
\text { P-value }\end{array}$ \\
\hline \multicolumn{5}{|l|}{ Static } \\
\hline $\mathrm{TBR}_{\max }$ & $\begin{array}{l}2.22 \\
{[1.36 ; 3.12]}\end{array}$ & $1.26[1.12 ; 1.37]$ & $2.71[2.20 ; 3.69]$ & $<.001$ \\
\hline $\mathrm{TBR}_{\text {mean }}$ & $\begin{array}{l}1.57 \\
{[1.02 ; 2.13]}\end{array}$ & $0.84[0.75 ; 1.04]$ & $1.95[1.57 ; 2.70]$ & $\begin{array}{l}<.001 \\
0.001\end{array}$ \\
\hline $\mathrm{TSR}_{\max }$ & $\begin{array}{l}1.31 \\
{[0.82 ; 1.90]}\end{array}$ & $0.77[0.65 ; 0.82]$ & $1.64[1.31 ; 2.28]$ & $<0.001$ \\
\hline $\mathrm{TSR}_{\text {mean }}$ & $\begin{array}{l}0.92 \\
{[0.65 ; 1.43]}\end{array}$ & $0.51[0.46 ; 0.65]$ & $1.19[0.92 ; 1.69]$ & $\begin{array}{l}< \\
0.001\end{array}$ \\
\hline MTV & $\begin{array}{l}1.49 \\
{[0.00 ; 8.22]}\end{array}$ & $0.00[0.00 ; 0.00]$ & 5.35 [1.49;15.75] & $\begin{array}{l}<.001 \\
0.001\end{array}$ \\
\hline \multicolumn{5}{|l|}{ Dynamic } \\
\hline TTP & $\begin{array}{l}7.70 \\
{[3.35 ; 18.65]}\end{array}$ & $14.53[1.55 ; 30.00]$ & $7.67[4.13 ; 14.62]$ & 0.92 \\
\hline slope & $\begin{array}{l}-0.14 \\
{[-0.82 ; 0.13]}\end{array}$ & $0.07[-0.07 ; 0.31]$ & $-0.59[-0.94 ;-0.08]$ & $\begin{array}{l}<.001 \\
0.00\end{array}$ \\
\hline
\end{tabular}


Table 3

Results provided by ROC curve analyses for the PET identification of patients with glioma recurrence or progression.

\begin{tabular}{|c|c|c|c|c|c|c|}
\hline & AUC & $\begin{array}{l}\mathrm{Cl}(95 \%) \\
\text { AUC }\end{array}$ & Threshold & Sensitivity & Specificity & Accuracy \\
\hline $\mathrm{TBR}_{\max }$ & 0.969 & $(0.923-1.0)$ & 1.61 & $97.1 \%$ & $94.1 \%$ & $96.0 \%$ \\
\hline $\mathrm{TBR}_{\text {mean }}$ & 0.983 & $(0.956-1.0)$ & 1.3 & $94.1 \%$ & $94.1 \%$ & $94.1 \%$ \\
\hline $\mathrm{TSR}_{\max }$ & 0.976 & $(0.939-1.0)$ & 1.0 & $97.1 \%$ & $94.1 \%$ & $96.0 \%$ \\
\hline $\mathrm{TSR}_{\text {mean }}$ & 0.986 & $(0.964-1.0)$ & 0.83 & $91.2 \%$ & $100 \%$ & $94.1 \%$ \\
\hline MTV & 0.978 & $(0.939-1.0)$ & $0.045 \mathrm{~mL}$ & $97.1 \%$ & $94.1 \%$ & $96.0 \%$ \\
\hline Slope & 0.818 & $(0.702-0.935)$ & $-0.26 h^{-1}$ & $67.6 \%$ & $94.1 \%$ & $76.5 \%$ \\
\hline \multicolumn{7}{|c|}{$\begin{array}{l}\text { AUC: area under the curve; Cl: confidence interval; MTV: metabolic tumor volume; TBR: tumor-to- } \\
\text { normal brain ratio; TSR: tumor-to-striatum ratio }\end{array}$} \\
\hline \multicolumn{7}{|c|}{$\begin{array}{l}\text { a. 51-year-old woman with no recurrent or progressive glioma }\left(\mathrm{TBR}_{\operatorname{mean}}=0.8, \mathrm{TBR}_{\max }=1.1 \text {, }\right. \\
\left.\mathrm{TSR}_{\text {mean }}=0.5, \mathrm{TSR}_{\max }=0.7, \mathrm{MTV}=0 \mathrm{~mL}, \mathrm{TTP}=30 \mathrm{~min} \text { and slope }=0.31 \mathrm{~h}^{-1}\right) \text {. }\end{array}$} \\
\hline \multicolumn{7}{|c|}{$\begin{array}{l}\text { b. 46-year-old woman with a progressive IDH-wildtype glioblastoma }\left(\mathrm{TBR}_{\text {mean }}=1.9, \mathrm{TBR}_{\mathrm{max}}=2.5 \text {, }\right. \\
\left.\mathrm{TSR}_{\text {mean }}=1.2, \mathrm{TSR}_{\max }=1.6, \mathrm{MTV}=6.13 \mathrm{~mL}, \mathrm{TTP}=7.6 \mathrm{~min} \text { and slope }=-1.22 \mathrm{~h}^{-1}\right) .\end{array}$} \\
\hline
\end{tabular}

On multivariate analysis, the sole best independent predictor of glioma recurrence/progression was $\mathrm{TSR}_{\max }$, with no other dynamic or static PET parameter able to provide any significant additional predictive information.

\section{PET parameters for predicting PFS and OS}

All PET parameters, except TTP, were also significant predictors of PFS on Kaplan-Meier analyses, although none were predictive of OS (adjusted $p>0.75$ ). More precisely, PFS times were much longer in patients with vs. those without a $\mathrm{TBR}_{\max } \leq 1.61$ (29.7 vs. 0.4 months; log-rank test adjusted $p<0.001$ ), a $\mathrm{TBR}_{\text {mean }} \leq 1.3$ (27.8 vs. 0.5 months; log-rank test adjusted $\left.\mathrm{p}<0.001\right)$, a TSR $\mathrm{Tax}_{\max } \leq 1.0$ (29.7 vs. 0.4 months; log-rank test adjusted $p<0.001)$, a TSR mean $\leq 0.083$ ( 25.6 vs. 0.0 months; log-rank test adjusted $p<0.001$ ), a MTV $\leq 0.045 \mathrm{~mL}$ (29.7 vs. 0.4 months; log-rank test adjusted $\mathrm{p}<0.001)$ or a curve slope $\geq-0.26 \mathrm{~h}^{-1}$ (18.0 vs. 0.4 months; log-rank test adjusted $p<0.001$ ). The corresponding survival curves are depicted in Fig. 1.

Representative examples of patients with or without glioma recurrence/progression investigated with ${ }^{18} \mathrm{~F}$ FDopa imaging are provided in Fig. 2. 


\section{Discussion}

In the present population of patients with suspected glioma recurrence/progression at post-resection MRI, both static and dynamic ${ }^{18} \mathrm{~F}$-FDopa PET parameters were significant predictors of a glioma recurrence/progression at 6-months, as well as of progression-free survival on the longer term. This diagnostic information was however mostly achieved with conventional static parameters, with limited additional diagnostic information provided by dynamic parameters, contrary to that previously reported in the very different clinical setting of newly-diagnosed gliomas [10,21].

The diagnostic performances of ${ }^{18} \mathrm{~F}$-FDopa PET imaging in the present study were very high, in keeping with those reported in a previous study [8], reaching a global accuracy of $96 \%$ for predicting patients with glioma recurrence/progression at 6-months after the ${ }^{18} \mathrm{~F}$-FDopa PET exam. This global accuracy is moreover higher when compared with the $82 \%$ level previously reported for the ${ }^{18} \mathrm{~F}$-FDopa PET detection of glioblastoma recurrence in a population of 110 patients [6] as well as with levels documented with other amino-acid PET tracers such as ${ }^{11} \mathrm{C}$-methyl-methionine (MET) [22-24] or ${ }^{18} \mathrm{FET}$-PET [25-28].

To the best of our knowledge, the present study is the first in which the detection of such recurrence/progression was tested with the added input of dynamic parameters to the conventional static ${ }^{18} \mathrm{~F}$-FDopa PET parameters and assessed in a large spectrum of glioma entities precisely characterized according to the WHO 2016 classification.

Of particular note, of the two studied dynamic parameters, only the curve slope was shown to provide significant predictability of glioma recurrence/progression, albeit with a relatively lower diagnostic accuracy than that provided by each of the static parameters (Table 3). Moreover, upon multivariate analysis, none of the dynamic parameters were shown to provide any additional diagnostic information, comparatively to that obtained with static parameters.

These results are at least partly supported by previous results documented for ${ }^{18} \mathrm{~F}-\mathrm{FET}$ and in which the univariate predictive values of the dynamic parameters were consistently found lower than that of static parameters [25-28]. In particular, in a large study cohort including 124 patients, dynamic ${ }^{18}$ FET-PET parameters (TTP, curve pattern) were less predictive than static parameters $\left(T B R_{\text {max }}, T R_{\text {mean }}\right)$ for glioma recurrence/progression [25].

The present study thus supports the general consideration that dynamic parameters from amino-acid PET imaging exhibit a lower performance in detecting gliomas after treatment than before treatment when considering a population composed of both low-grade and high-grade gliomas. This statement could be moderated regards to the article of Fleischmann et al., which shows that ${ }^{18} \mathrm{FET}$-PET dynamic parameters have a higher prognostic value in tumor recurrences than static parameters in a series of high-grade gliomas [29]. This latter hypothesis is further underlined in the present study by our additional observation whereby, when separating gliomas into two groups according to their respective grade at initial diagnosis [30], all of the static parameters as well as curve slope were able to discriminate recurrent 
or progressive gliomas in initially classified high-grade gliomas $(\mathrm{p} \leq 0.001)$ whereas such was not the case for the slope parameter in low-grade gliomas $(p=0.13)$ (Supplemental Figure). This statement is clinically pertinent since detecting recurrence/progression can be more challenging in high-grade gliomas as compared to low-grade gliomas given the more frequent use of radiotherapy, and thus to the occurrence of potential post-radiotherapeutic changes $[13,14]$. Despite the fact that the number of patients was relatively limited, particularly for low-grade gliomas, this finding further strengthens the fact that dynamic parameters must be more relevant in instances of high expected uptake, i.e. in high grade gliomas [3-5], in which a clear decreasing slope is typically reported in these most aggressive gliomas entities $[9,10]$. By contrast, lower-grade gliomas preferentially exhibit consistently increasing curves [10, 31,32 , which is very similar to ${ }^{18} \mathrm{~F}$-FDopa time-activity curves expected in normal brain tissue or reactive tissue changes [3]. The use of dynamic parameters in this setting of low-grade gliomas could be then more challenging. Another potential rationale is that a sufficiently high tumor uptake is required for an accurate determination of dynamic parameters, whereby this uptake was too low in the present study in instances of an absence of any glioma recurrence/progression (median $\mathrm{TBR}_{\text {mean }}=0.84$; see Table 2). In contrast to the previous hypotheses, the potential confounding influence of reactive tissue changes, in line with the inflammatory- and healing-process induced by surgery and/or radiotherapy couldn't be used to explain the lower diagnostic performances from dynamic parameters. Static and dynamic parameters should be both affected in the same way by the complex histology of the treated sites, involving varying levels of reactive gliosis, inflammatory cells and radiation-induced. Moreover, in our clinical practice, ${ }^{18} \mathrm{~F}$ FDopa PET for detecting glioma recurrence/progression are planned at least 2 months after the surgery or the end of the radiotherapy, limiting the risk of the previously enounced confounding factors.

${ }^{18} \mathrm{~F}$-FDopa PET imaging was also predictive of PFS in this series of suspected recurrent gliomas, with a lower uptake associated with a longer PFS. Several studies have observed similar relationships between static amino-acid PET parameters and PFS in recurrent gliomas [6, 33-36]. Of note, the study by Herrmann and al. also showed that ${ }^{18} \mathrm{~F}$-FDopa PET imaging was able to predict PFS but not OS [6], as observed in the current study. Concerning dynamic parameters, two studies with ${ }^{18}$ FET-PET report their predictive value on survival with longer survival observed in cases of consistently increasing curves or short time-to-peak [29,36], an observation corroborated by the present results in which an increasing slope was associated with a longer PFS (Fig. 1). Irrespectively, the data provided herein by ${ }^{18} \mathrm{~F}$-FDopa PET imaging was furthermore predictive of patient outcome well beyond the 6-month period, reaching up to 18 and 29 months of mean PFS according to dynamic or static parameters, respectively.

The main limitations inherent to this study are that results were obtained retrospectively, in a single center. In addition, our sample size was too limited for providing separate analyses according to the different molecular features of the gliomas involved in the present study, whereas ${ }^{18} \mathrm{~F}$-FDopa uptake is known varying according to these features [37]. These findings hence need to be confirmed in further multicenter prospective studies planned in larger populations. Furthermore, the relative low number of histologically verified cases should lead to consider with caution the very high accuracy reported in this study.

Page $11 / 19$ 
The time activity curves of tumors were expressed through ratios with activity from normal brain, a method relatively uncommon in this setting. However, this method was successfully tested by our team for the detection of newly-diagnosed gliomas [10], along with the necessity to lower the possible interference of Carbidopa premedication taken by certain patients [38]. Lastly, the calculation of the PFS and OS may be questioned given that all included gliomas, mixing several entities and especially lowand high-grade gliomas, may have benefited from different therapeutic strategies. Notwithstanding, all applied treatments were performed in keeping with general standards [13, 14].

\section{Conclusion}

In summary, this novel study, assessing the relevance of ${ }^{18} \mathrm{~F}-\mathrm{FD}$ opa kinetics in the diagnosis of recurrent gliomas for the first time, shows that patients with a glioma recurrence/progression, occurring remotely after surgery, may be detected by both static and dynamic ${ }^{18} \mathrm{~F}$-FDopa PET parameters. However, in this population mixing both low- and high-grade gliomas, most of this diagnostic information is achievable by conventional static parameters, contrary to that previously documented for the ${ }^{18} \mathrm{~F}$-FDopa PET detection of newly-diagnosed gliomas. Further studies should be planned to investigate the relevance of such ${ }^{18} \mathrm{~F}-$ FDopa kinetics in population involving only high-grade gliomas.

\section{Abbreviations}

PFS

Progression-Free Survival

RANO

Response Assessment in Neuro-Oncology

EANM

European Association of Nuclear Medicine

EANO

European Association of Neuro-oncology

WHO

World Health Organization

OS

Overall Survival

CT

Computed Tomography

ROI

Region Of Interest

SUV

Standardized Uptake Values

TBR

Tumor-to-normal-Brain Ratio 
TSR

Tumor-to-Striatum Ratio

FLAIR

Fluid Attenuation Inversion Recovery

MTV

Metabolic Tumor Volume

TTP

Time-To-Peak

GBM

Glioblastomas

MET

${ }^{11} \mathrm{C}$-methyl-methionine

\section{Declarations}

Ethics approval and consent to participate: All procedures performed in studies involving human participants were in accordance with the ethical standards of the institutional and/or national research committee and with the 1964 Helsinki declaration and its later amendments or comparable ethical standards. Written informed consent was obtained from all individual participants included in the study. The local ethics committee (Comité d'Ethique du CHRU de Nancy) approved the retrospective data evaluation on June 7, 2018, and authorization from the CNIL (National Commission on Information Technology and Civil Liberties) was delivered on June 25, 2018 (authorization n R2018-11).

Consent for publication: Consent to publish has been obtained for all individual person's data included in this manuscript.

Availability of data and materials: All data generated or analyzed during this study are included in this published article and its supplementary information files.

Competing interests: All authors declare that they have no competing interests.

Funding: Not applicable

Authors' contributions: All authors participated either to: 1) study conception and design or analysis and interpretation of the data, or both (TZ, MG, VR, ZL, AV); 2) the drafting of the manuscript or revising it critically for important intellectual content (TZ, MG, FR, MB, GG, PYM, LI, AV) or 3) final approval of the submitted manuscript (FR, MB, GG, LT, LI, AV).

Acknowledgements: We gratefully acknowledge the excellent technical supports of PET radiological technologist at our institution. 


\section{References}

1.

Albert NL, Weller M, Suchorska B, Galldiks N, Soffietti R, Kim MM, et al. Response Assessment in NeuroOncology working group and European Association for Neuro-Oncology recommendations for the clinical use of PET imaging in gliomas. Neuro-Oncol. 2016;18:1199-208.

2.

Law I, Albert NL, Arbizu J, Boellaard R, Drzezga A, Galldiks N, et al. Joint EANM/EANO/RANO practice guidelines/SNMMI procedure standards for imaging of gliomas using PET with radiolabelled amino acids and [18F]FDG: version 1.0. Eur J Nucl Med Mol Imaging. 2019;46:540-57.

3.

Chen W, Silverman DHS, Delaloye S, Czernin J, Kamdar N, Pope W, et al. 18F-FDOPA PET imaging of brain tumors: comparison study with 18F-FDG PET and evaluation of diagnostic accuracy. J Nucl Med. 2006;47:904-11.

4.

Fueger BJ, Czernin J, Cloughesy T, Silverman DH, Geist CL, Walter MA, et al. Correlation of 6-18F-fluoro-Ldopa PET uptake with proliferation and tumor grade in newly diagnosed and recurrent gliomas. J Nucl Med. 2010;51:1532-8.

5.

Janvier L, Olivier P, Blonski M, Morel O, Vignaud J-M, Karcher G, et al. Correlation of SUV-Derived Indices With Tumoral Aggressiveness of Gliomas in Static 18F-FDOPA PET: Use in Clinical Practice. Clin Nucl Med. 2015;40:e429-35.

6.

Herrmann K, Czernin J, Cloughesy T, Lai A, Pomykala KL, Benz MR, et al. Comparison of visual and semiquantitative analysis of 18F-FDOPA-PET/CT for recurrence detection in glioblastoma patients. Neuro-Oncol. 2014;16:603-9.

7.

Youland RS, Pafundi DH, Brinkmann DH, Lowe VJ, Morris JM, Kemp BJ, et al. Prospective trial evaluating the sensitivity and specificity of 3,4-dihydroxy-6-[18F]-fluoro-L-phenylalanine (18F-DOPA) PET and MRI in patients with recurrent gliomas. J Neurooncol. 2018;137:583-91.

8.

Karunanithi S, Sharma P, Kumar A, Khangembam BC, Bandopadhyaya GP, Kumar R, et al. 18F-FDOPA PET/CT for detection of recurrence in patients with glioma: prospective comparison with 18F-FDG PET/CT. Eur J Nucl Med Mol Imaging. 2013;40:1025-35.

9.

Schiepers C, Chen W, Cloughesy T, Dahlbom M, Huang S-C.. 18F-FDOPA kinetics in brain tumors. J Nucl Med. 2007;48:1651-61. 
10.

Ginet M, Zaragori T, Marie P-Y, Roch V, Gauchotte G, Rech F, et al. Integration of dynamic parameters in the analysis of 18F-FDopa PET imaging improves the prediction of molecular features of gliomas. Eur $\mathrm{J}$ Nucl Med Mol Imaging. 2019.

11.

Sala Q, Metellus P, Taieb D, Kaphan E, Figarella-Branger D, Guedj E. 18F-DOPA, a clinically available PET tracer to study brain inflammation? Clin Nucl Med. 2014;39:e283-5.

12.

Wen PY, Macdonald DR, Reardon DA, Cloughesy TF, Sorensen AG, Galanis E, et al. Updated Response Assessment Criteria for High-Grade Gliomas: Response Assessment in Neuro-Oncology Working Group. J Clin Oncol. 2010;28:1963-72.

13.

Weller M, van den Bent M, Hopkins K, Tonn JC, Stupp R, Falini A, et al. EANO guideline for the diagnosis and treatment of anaplastic gliomas and glioblastoma. Lancet Oncol. 2014;15:e395-403.

14.

Weller M, van den Bent M, Tonn JC, Stupp R, Preusser M, Cohen-Jonathan-Moyal E, et al. European Association for Neuro-Oncology (EANO) guideline on the diagnosis and treatment of adult astrocytic and oligodendroglial gliomas. Lancet Oncol. 2017;18:e315-29.

15.

Kratochwil C, Combs SE, Leotta K, Afshar-Oromieh A, Rieken S, Debus J, et al. Intra-individual comparison of 18F-FET and 18F-DOPA in PET imaging of recurrent brain tumors. Neuro-Oncol. 2014;16:434-40. 16.

Schwarzenberg J, Czernin J, Cloughesy TF, Ellingson BM, Pope WB, Grogan T, et al. Treatment Response Evaluation Using 18F-FDOPA PET in Patients with Recurrent Malignant Glioma on Bevacizumab Therapy. Clin Cancer Res. 2014;20:3550-9.

17.

Ye H, Wong K-P, Wardak M, Dahlbom M, Kepe V, Barrio JR, et al. Automated movement correction for dynamic PET/CT images: evaluation with phantom and patient data. PloS One. 2014;9:e103745. 18.

Ceccon G, Lohmann P, Stoffels G, Judov N, Filss CP, Rapp M, et al. Dynamic O-(2-18F-fluoroethyl)-Ltyrosine positron emission tomography differentiates brain metastasis recurrence from radiation injury after radiotherapy. Neuro-Oncol. 2016.

19.

Benjamini Y, Hochberg Y. Controlling the False Discovery Rate: A Practical and Powerful Approach to Multiple Testing. J R Stat Soc Ser B Methodol. 1995;57:289-300.

20.

Louis DN, Perry A, Reifenberger G, von Deimling A, Figarella-Branger D, Cavenee WK, et al. The 2016 World Health Organization Classification of Tumors of the Central Nervous System: a summary. Acta Neuropathol (Berl). 2016;131:803-20.

21. 
Zaragori T, Guedj E, Verger A. Is IDH mutation status associated with 18 F-FDopa PET uptake? Ann Nucl Med. 2020;1-2.

22.

Terakawa Y, Tsuyuguchi N, Iwai Y, Yamanaka K, Higashiyama S, Takami T, et al. Diagnostic accuracy of $11 \mathrm{C}$-methionine PET for differentiation of recurrent brain tumors from radiation necrosis after radiotherapy. J Nucl Med. 2008;49:694-9.

23.

D'Souza MM, Sharma R, Jaimini A, Panwar P, Saw S, Kaur P, et al. 11C-MET PET/CT and advanced MRI in the evaluation of tumor recurrence in high-grade gliomas. Clin Nucl Med. 2014;39:791-8.

24.

Deuschl C, Kirchner J, Poeppel TD, Schaarschmidt B, Kebir S, El Hindy N, et al. 11C-MET PET/MRI for detection of recurrent glioma. Eur J Nucl Med Mol Imaging. 2018;45:593-601.

25.

Galldiks N, Stoffels G, Filss C, Rapp M, Blau T, Tscherpel C, et al. The use of dynamic O-(2-18Ffluoroethyl)-I-tyrosine PET in the diagnosis of patients with progressive and recurrent glioma. NeuroOncol. 2015;17:1293-300.

26.

Verger A, Filss CP, Lohmann P, Stoffels G, Sabel M, Wittsack H-J, et al. Comparison of O-(2-18FFluoroethyl)-L-Tyrosine Positron Emission Tomography and Perfusion-Weighted Magnetic Resonance Imaging in the Diagnosis of Patients with Progressive and Recurrent Glioma: A Hybrid Positron Emission Tomography/Magnetic Resonance Study. World Neurosurg. 2018;113:e727-37.

27.

Pyka T, Hiob D, Preibisch C, Gempt J, Wiestler B, Schlegel J, et al. Diagnosis of glioma recurrence using multiparametric dynamic 18F-fluoroethyl-tyrosine PET-MRI. Eur J Radiol. 2018;103:32-7.

28.

Maurer GD, Brucker DP, Stoffels G, Filipski K, Filss CP, Mottaghy FM, et al. 18F-FET PET imaging in differentiating glioma progression from treatment-related changes - a single-center experience. $\mathrm{J}$ Nucl Med. 2019.

29.

Fleischmann DF, Unterrainer M, Bartenstein P, Belka C, Albert NL, Niyazi M. 18F-FET PET prior to recurrent high-grade glioma re-irradiation-additional prognostic value of dynamic time-to-peak analysis and early static summation images? J Neurooncol. 2017;132:277-86.

30.

Louis DN, Ohgaki H, Wiestler OD, Cavenee WK, Burger PC, Jouvet A, et al. The 2007 WHO classification of tumours of the central nervous system. Acta Neuropathol (Berl). 2007;114:97-109. 31.

Pöpperl G, Kreth FW, Mehrkens JH, Herms J, Seelos K, Koch W, et al. FET PET for the evaluation of untreated gliomas: correlation of FET uptake and uptake kinetics with tumour grading. Eur $\mathrm{J}$ Nucl Med Mol Imaging. 2007;34:1933-42.

32. 
Lohmann P, Herzog H, Rota Kops E, Stoffels G, Judov N, Filss C, et al. Dual-time-point O-(2-

[(18)F]fluoroethyl)-L-tyrosine PET for grading of cerebral gliomas. Eur Radiol. 2015;25:3017-24.

33.

Beppu T, Terasaki K, Sasaki T, Sato Y, Tomabechi M, Kato K, et al. MRI and 11C-methyl-L-methionine PET Differentiate Bevacizumab True Responders After Initiating Therapy for Recurrent Glioblastoma. Clin Nucl Med. 2016;41:852-7.

34.

Beppu T, Sato Y, Sasaki T, Terasaki K, Yamashita F, Sasaki M, et al. Comparisons Between PET With 11CMethyl-L-Methionine and Arterial Spin Labeling Perfusion Imaging in Recurrent Glioblastomas Treated With Bevacizumab. Clin Nucl Med. 2019;44:186-93.

35.

Hutterer M, Nowosielski M, Putzer D, Waitz D, Tinkhauser G, Kostron H, et al. O-(2-18F-fluoroethyl)-Ltyrosine PET predicts failure of antiangiogenic treatment in patients with recurrent high-grade glioma. $J$ Nucl Med. 2011;52:856-64.

36.

Galldiks N, Rapp M, Stoffels G, Fink GR, Shah NJ, Coenen HH, et al. Response assessment of bevacizumab in patients with recurrent malignant glioma using [18F]Fluoroethyl-L-tyrosine PET in comparison to MRI. Eur J Nucl Med Mol Imaging. 2013;40:22-33.

37.

Verger A, Metellus Ph, Sala Q, Colin C, Bialecki E, Taieb D, et al. IDH mutation is paradoxically associated with higher 18F-FDOPA PET uptake in diffuse grade II and grade III gliomas. Eur J Nucl Med Mol Imaging. 2017;44:1306-11.

38.

Hoffman JM, Melega WP, Hawk TC, Grafton SC, Luxen A, Mahoney DK, et al. The effects of carbidopa administration on 6-[18F]fluoro-L-dopa kinetics in positron emission tomography. J Nucl Med. 1992;33:1472-7.

\section{Figures}


$\mathrm{TBR}_{\max }$

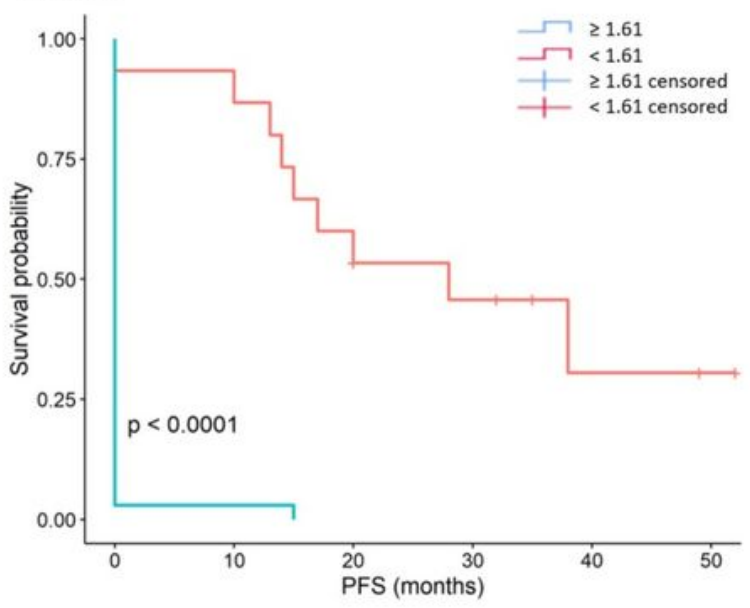

$T_{B R}$ mean

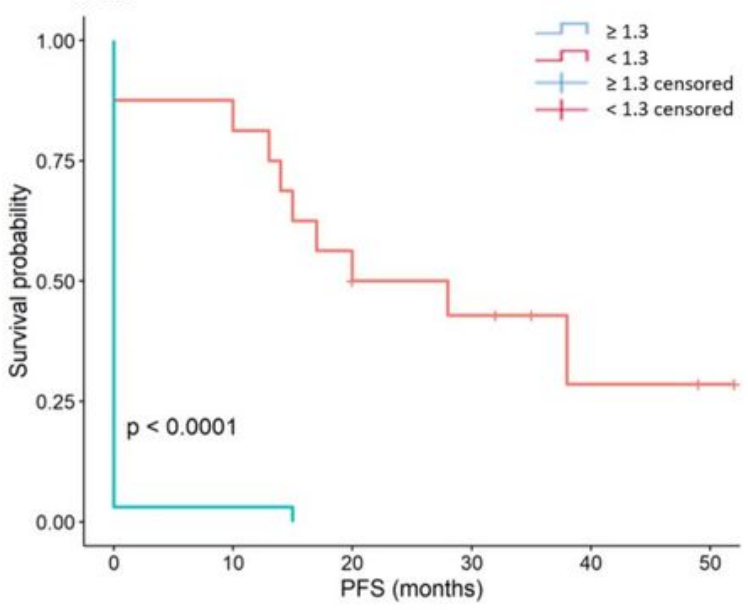

MTV

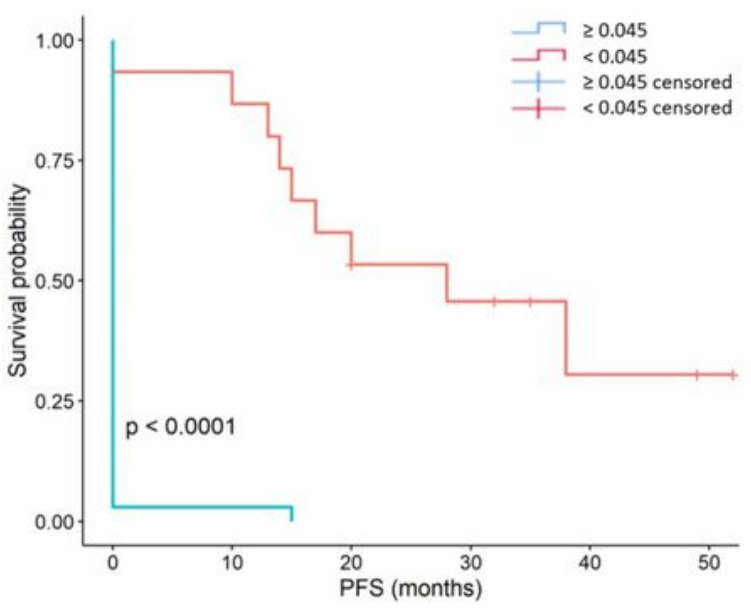

$\mathrm{TSR}_{\max }$

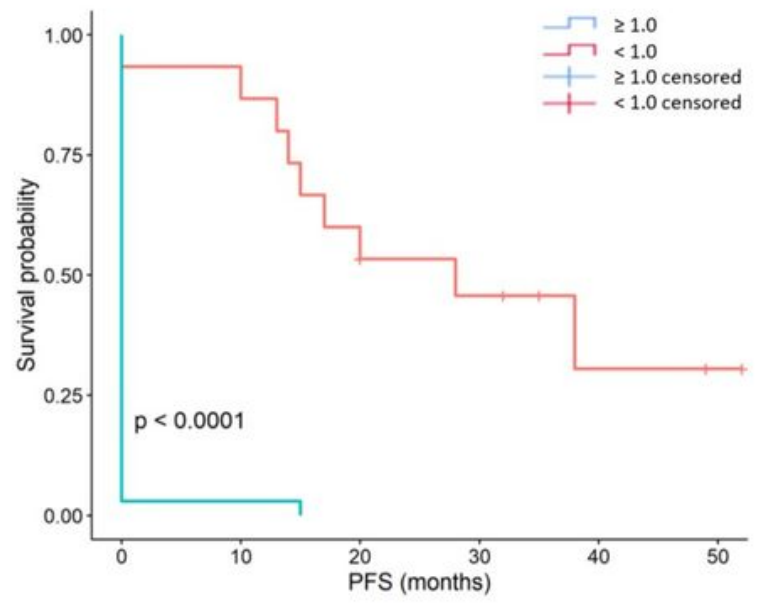

$\mathrm{TSR}_{\text {mean }}$

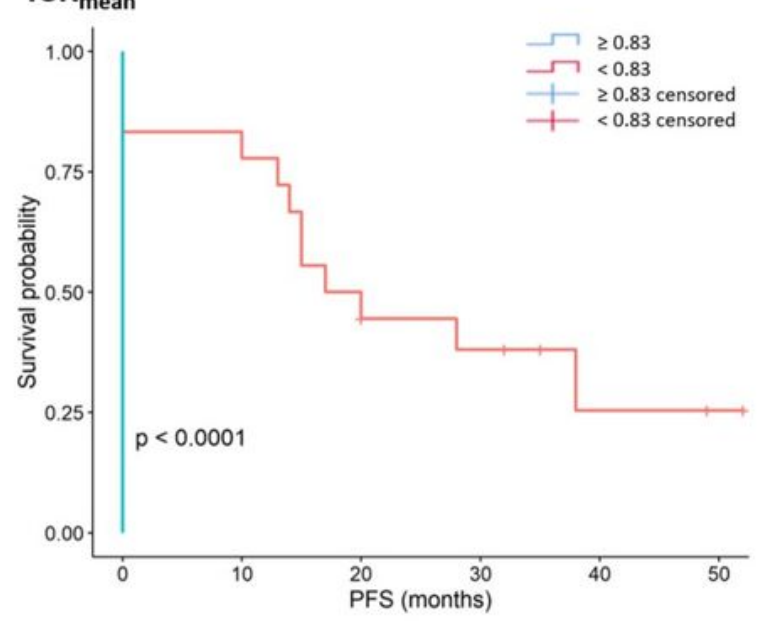

Slope

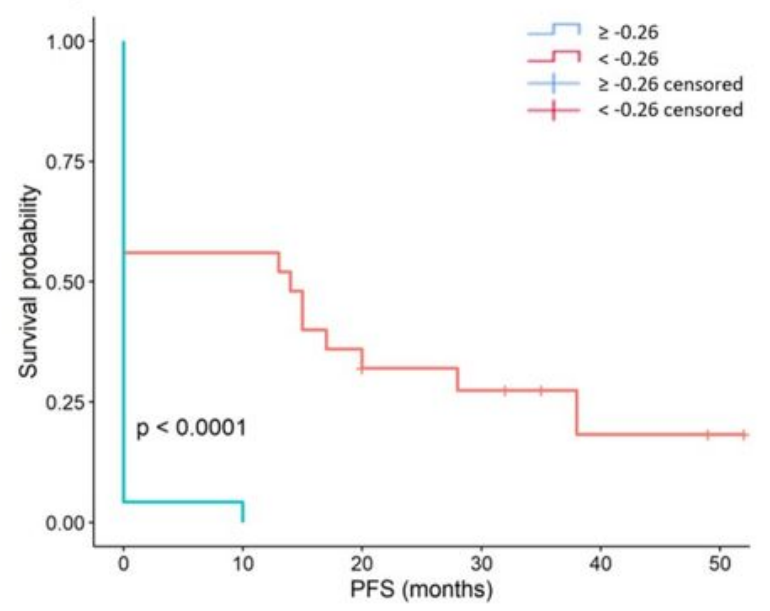

\section{Figure 1}

Kaplan-Meier survival plots for the prediction of progression-free survival using maximal tumor-tobackground ratios (upper left panel), mean tumor-to-background ratios (middle left panel), metabolic tumor volume (lower left panel), maximal tumor-to-striatum ratio (upper right panel), mean tumor-tostriatum ratio (middle right panel) and slope (lower right panel) as discriminators. Corresponding log-rank test adjusted $p$ values are $<0.0001$ for all presented curves. 


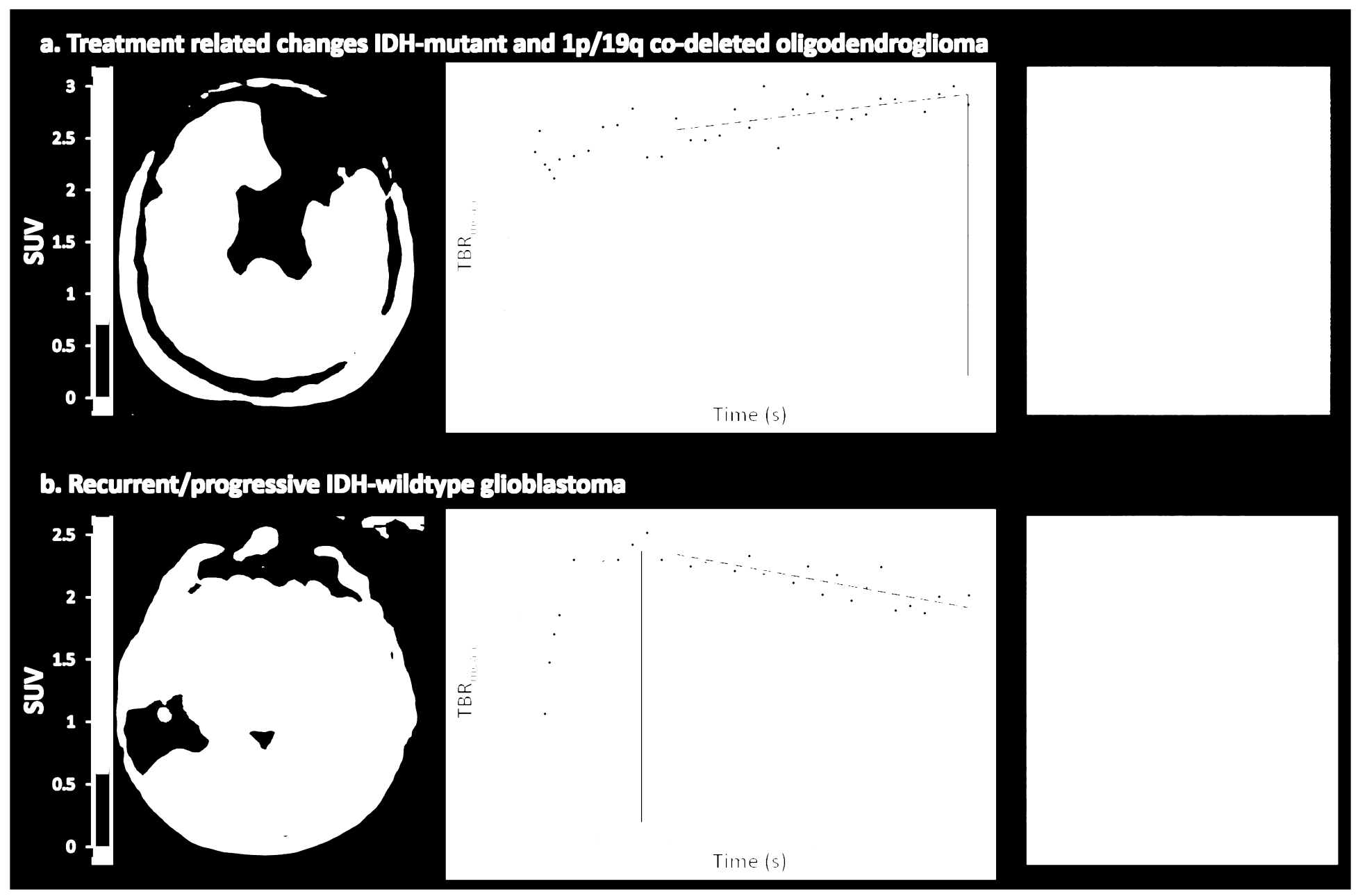

Figure 2

Representative examples of patients with or without glioma recurrence/progression investigated with 18F-FDopa imaging, with axial slices of 18F-FDopa PET (left column), dynamic TBRmean curves (middle column) providing the time-to-peak delay-time (light blue dotted line) and the 10-to-30 min slope (dark blue dotted line), along with, for illustrative purposes, the same slice location recorded on a FLAIR MRI sequence (right column). a. 51-year-old woman with no recurrent or progressive glioma (TBRmean $=0.8$, TBRmax =1.1, TSRmean=0.5, TSRmax=0.7, MTV=0 mL, TTP=30 min and slope=0.31 h-1). b. 46-year-old woman with a progressive IDH-wildtype glioblastoma (TBRmean=1.9, TBRmax $=2.5$, TSRmean=1.2, TSRmax=1.6, MTV=6.13 mL, TTP=7.6 min and slope=-1.22 h-1).

\section{Supplementary Files}

This is a list of supplementary files associated with this preprint. Click to download.

- SupplementalFigureHD.tiff

- SupplementalFigure.docx

- SupplementalMaterial.xlsx 